\title{
Infrared optical imaging of matrix metalloproteinases (MMPs) up regulation following ischemia reperfusion is ameliorated by hypothermia
}

\author{
Philip A Barber ${ }^{1,3^{*}}$, David Rushforth², Smriti Agrawal ${ }^{1}$ and Ursula I Tuor ${ }^{1,2,3}$
}

\begin{abstract}
Background: We investigated the use of a new MMP activatable probe MMPSense 750 FAST (MMPSense750) for in-vivo visualization of early MMP activity in ischemic stroke. Following middle cerebral artery occlusion (MCAO) optical imaging was performed. Near-infrared (NIR) fluorescent images of MMPSense activation were acquired using an Olympus fluorescent microscope, 1.25x objective, a CCD camera and an appropriate filter cube for detecting the activated probe with peak excitation and emission at 749 and $775 \mathrm{~nm}$, respectively. Images were acquired starting at 2 or 24 hours after reperfusion over the ipsilateral and contralateral cortex before and for 3 hours after, MMPSense750 was injected.
\end{abstract}

Results: Increased intensities ipsilaterally were observed following MMPSense750 injection with ischemic injury but not in sham animals. There were significant ipsilateral and contralateral differences at 15 minutes $(P<0.05)$ in early ischemic reperfusion and at time 0 in 24 hours post ischemia $(P<0.05)$ which persisted at 180 minutes in both these groups $(P<0.01)$, but not following sham surgery. The increase in ipsilateral signal intensity was attenuated by hypothermia. These observations corresponded with a significant increase in the total MMP-9 protein levels, 5 and 24 hours following ischemia reperfusion $(P<0.05)$ and their reduction by hypothermia.

Conclusions: Matrix-metalloproteinase upregulation in ischemia reperfusion can be imaged acutely in-vivo with NIRF using MMPSense750. Hypothermia attenuated both the optical increase in intensity after MMPSense750 and the increase in MMP-9 protein expression supporting the proof of concept that NIRF imaging using MMPSense can be used to assess potential therapeutic strategies for stroke treatment.

\section{Background}

Stroke is the third leading cause of death and the leading cause of long-term disability in adults. Several members of the matrix metalloproteinase (MMPs) family have been implicated to have detrimental roles in stroke [1,2]. Specifically, the gelatinases MMP-2 and MMP-9 have previously been considered to specifically injure the important components of the basal lamina around the cerebral blood vessels that precede microvascular

\footnotetext{
* Correspondence: pabarber@ucalgary.ca

'Department of Clinical Neurosciences, Experimental Imaging Centre and Hotchkiss Brain Institute, Faculty of Medicine, University of Calgary, Calgary, Canada

${ }^{3}$ Experimental Imaging Centre, B153, 3330 Hospital Dr NW, University of Calgary, Calgary, AB T2N 2T9, Canada

Full list of author information is available at the end of the article
}

damage in cerebral ischemia [1]. This leads to disruption of the blood brain barrier, edema, and hemorrhagic transformation in animal models of ischemia $[3,4]$. Once within the CNS, MMPs continue to damage CNS tissue. In general higher MMP-9 levels are shown to correlate significantly with larger infarct volume, severity of stroke, reduced survival of neurons and worse functional outcome [5], and therefore, seem an appropriate target for a robust neuroprotectant such as hypothermia $[6,7]$.

Most current studies investigating cerebral ischemia and stroke detect MMPs in vitro using techniques such as Western Blotting [8], ELISA [9] RT-PCR or staining with anti-MMP antibodies [10]. While these are good techniques they fall short when reporting accurate levels of the active form of MMP, are limited in their

\section{() Biomed Central}


sensitivities of MMP detection, and may not be a precise depiction of MMP activity in vivo. In vivo visualization of MMP activity would provide important information regarding the spatial and temporal expression of MMP enzymatic activity with respect to the pathophysiology of the disease and to monitor response to interventions, such as therapeutic hypothermia.

NIRF emitting probes offer the advantage of increased depth of detection. Recently probes that fluoresce upon proteolytic cleavage by MMP's have become available with the potential advantage of exhibiting low background fluorescence. One such probe, MMPSense680 (Perkin Elmer Inc (previously VisEn Medical), Boston, MA USA) with a peak excitation at approx $680 \mathrm{~nm}$ and emission at $700 \mathrm{~nm}$, was shown to produce increased fluorescent signal considered related to MMP mediated cleavage in diseases including stroke $[11,12]$. Activation of the probe is reported to occur by a broad range of MMP's including MMP 2, 3, 9, and 13 [11] and the manufacturer recommends imaging at $24 \mathrm{hr}$ following administration.

The objective of the present study was to determine whether an alternate MMP activatable probe with a shorter optical imaging time, MMPSense 750 FAST (MMPSense750), could be used for visualization of MMP activity in the early stages of ischemia reperfusion in a mouse model of stroke. This probe also has fluorescent properties in the near infrared range (peak excitation and emission at approx $749 \mathrm{~nm}$ and $775 \mathrm{~nm}$, respectively) and according to the manufacturer is sensitive to cleavage and optical activation by various MMP's including MMP2,3,7,9,12 and MMP13. In order to investigate the responsiveness of the probe to alterations in MMP activity, the current study also examined whether the MMPSense750 probe would detect reductions in MMP's associated with a therapeutic intervention following stroke We chose mild hypothermia for its well documented therapeutic reductions in various MMPs in a number of neurological diseases including stroke [13-16]. In parallel with in vivo studies, characteristics of the probe were investigated in vitro.

\section{Methods}

\section{Optical activation of MMPSense750 in vitro}

To test the optical changes in MMPSense750 (Elmer Perkin Inc, Boston MA, USA) in vitro, we investigated fluorescent intensity changes following its addition to several different test solutions. The test solutions consisted of blood (from an animal 2 hours following MCAO plus reperfusion), phosphate buffer (PBS), and PBS with an enzyme (tryspin, $1 \mathrm{mg} / \mathrm{ml}$ ) that would cleave and activate the optical probe. The test solution (95ul) was added to a 96 well plate and a zero reading was taken. Then 5ul of MMPSense750, was added to the wells and shaken. Near-Infrared Images were acquired repeatedly over 30 minutes from which intensity changes were measured. In control experiments, PBS was added instead of MMPSense750. Experiments were repeated in duplicate or triplicate. .

\section{Near infrared fluorescent imaging}

Near-Infrared images were acquired using a $1.25 \times$ objective (PLAN-APO) on an Olympus BX51 fluorescent microscope fitted with a back thinned CCD camera (Qimaging Rolera) and an Olympus Cy7-B-OMF-ZERO filter cube with excitation/emission bands of 665-750 nm and 765 to $855 \mathrm{~nm}$, respectively. Digital images (14 bit monochromatic) were acquired with a temporal resolution of $4 \mathrm{sec}$ and a pixel size of $12.6 \mu \mathrm{mx} 12.6 \mu \mathrm{m}$ $(512 \times 512$ pixels $)$. Emission intensity was measured as mean gray levels in regions of interest using Image-J software (Research Services Branch, National Institute of Mental Health, Bethesda, Maryland, USA).

\section{In vivo experiments}

Male C57Bl/6 mice (3 months old, 25 to 35 g; Charles River Breeding Laboratories, Ontario, Canada) were prepared for transient MCAO using the intraluminal filament method [17]. A total of forty-six animals were included in the study. Of these 25 were dedicated for optical imaging and 21 for quantification of MMP protein expression with ELISA. The animal groups were SHAM $(\mathrm{N}=13)$, early ischemia reperfusion $(\mathrm{N}=14)$, early ischemia reperfusion with the induction of hypothermia (33 C) $(\mathrm{N}=8)$, and ischemia reperfusion at 24 hours $(\mathrm{N}=11)$. All experiments and procedures were approved by the local University of Calgary and National Research Council animal care committees and were in accordance with the Canadian Council of Animal Care guidelines.

\section{Transient focal ischemia}

Anesthesia was induced with isoflurane (3\% initial, 1\% to $1.5 \%$ maintenance) in $\mathrm{O}_{2}$ and air (80\%:20\%). Briefly, under the operating microscope, the left common carotid artery (CCA), the left external carotid artery (ECA), and the left internal carotid artery (ICA) were isolated and a 6-0 suture was tied at the origin of the ECA and at the distal end of the ECA. The left CCA and ICA were temporarily occluded. The silicon-coated nylon suture (diameter 180-220 $\mu \mathrm{m}$ ) was introduced into the ECA and inserted into the ICA approximately 9 to $10 \mathrm{~mm}$ from the carotid bifurcation until meeting resistance and effectively blocking the middle cerebral artery. The suture remained inserted for 30 minutes, after which it was removed and the ECA was permanently tied. 


\section{Measurement of cerebral blood flow}

Transcranial measurements of cerebral blood flow (CBF) were made by laser-Doppler flowmetry (LDF) while the animal was under general anesthesia (Perisoft Version 1.3; Perimed Inc). A $0.5-\mathrm{mm}$ diameter microfiber laserDoppler probe (Probe 418; Perimed) was attached to the skull with cyanoacrylate glue $6 \mathrm{~mm}$ lateral and $1 \mathrm{~mm}$ posterior of bregma. Data were expressed as a mean percentage of the baseline pre-ischemia value. The occlusion was considered adequate if $\geq 70 \%$ reduction in cortical CBF occurred immediately after placement of the intraluminal occluding suture; otherwise, mice were excluded.

\section{Temperature regulation}

Mice were implanted with intra-abdominal radiofrequency probes (TA10TA-F20; Transoma Medical) 7 days before MCA occlusion. Core temperature was sampled every 30 seconds using receivers Activity and temperature data was collected every 30 seconds during movement of the animal over the receiver (RLA-1020; Data Sciences Int.) interfaced to a computer running ART 2.2. During the surgical procedure, the animals were regulated at $36.5^{\circ} \mathrm{C}$ (total time 2 hours) by a $125-\mathrm{W}$ heating lamp.

\section{Intravital fluorescence measurements}

Ninety minutes or 24 hours post-reperfusion, a lateral tail vein was cannulated for intravenous infusion of MMPSense750. Core body temperature was continuously monitored and maintained using a rectal probe with feedback to a heating lamp for the duration of the experiment at $36.5{ }^{\circ} \mathrm{C}$ for normothermia or $33.5{ }^{\circ} \mathrm{C}$ for hypothermia (started at the onset of reperfusion and maintained for the duration of the experiment). Because initial experiments had difficulty imaging MMPSense 750 through the skull we created a window using a highspeed micro-drill and saline cooling to expose the left parietal bone between the lambdoidal suture, sagittal suture and coronal suture. A control window was made on the contralateral side.

Animals were placed on a custom designed heated platform attached to the stage of the Olympus BX51 microscope. One hundred and twenty minutes after reperfusion, animals were imaged over ipsilateral and contralateral parietal cortex to obtain baseline background measures and then $150 \mu \mathrm{l}$ MMPSense750 was infused over 60 seconds. Optical imaging data was then acquired every 5-10 minutes over 3 hours. At 3 hours animals were euthanized and the entire brain was removed and imaged ex-vivo using a FITC filter cube (maximal excitation and emission of 490 and $520 \mathrm{~nm}$, respectively) instead of the NIR filter cube. Endogenous blue/green fluorescence was increased in the ischemic hemisphere and this fluorescence was considered related to increased levels of mitochondrial coenzymes (e.g.
NADH and NADPH) [18] produced within the ischemic lesion[19] thereby providing an estimate of the extent of the stroke. Brains were dissected and prepared for histology or ELISA assay of MMP-9 and 2.

\section{ELISA assay}

Brains were cut into thick axial sections in PBS at $4{ }^{\circ} \mathrm{C}$. Stroke lesion was identified by the endogenous region of autofluorescence with FITC imaging and a sample was dissected along with an equivalent sample in the contralateral region. Tissue was homogenized in radioimmunoprecipitation assay (RIPA) buffer with Ethylenediaminetetraacetic acid (EDTA)-free protease inhibitors (Roche) by sonication for 2 minutes on ice. Homogenates were centrifuged ( 1000 G, 5 mins) and supernatants collected for analysis. ELISA kits were used according to the manufacturer's instructions for measurement of murine total and pro- murine MMP-9, (Quantikine ELISA Kits,R \& D systems) and murine MMP-2 (Abnova). Supernatants were assayed for protein concentration (Pierce, BCA assay kit) and results were expressed as ng MMP per mg total protein.

\section{Histology}

Animals were euthanized with sodium pentobarbital (70 $\mathrm{mg} / \mathrm{kg}$ intraperitoneally) and transcardially perfused with $0.9 \%$ saline, followed by $4 \%$ paraformaldehyde. The brain was then embedded in paraffin and sections were cut at $6 \mu \mathrm{m}$ thickness and stained with hematoxylin and eosin. The distance between sections was $\approx 1 \mathrm{~mm}$. Paraffin sections were de-waxed in xylene and rehydrated. Antigen retrieval was performed by boiling sections in citrate buffer for 2 minutes. Sections were then washed in PBS (0.01 M),Triton X100(1\%) for 10 minutes followed by 2 subsequent washes in $\operatorname{PBS}(0.01 \mathrm{M})$ for 5 mins. Normal goat serum $(10 \%) / \mathrm{PBS}(0.01 \mathrm{M})$ was then applied to the sections for 30 minutes at room temperature to block non-specific binding. Primary antibody (MAB305, Millipore) Rabbit anti MMP-9 n-terminal was diluted 1/200 in PBS $(0.01 \mathrm{M}) / \mathrm{BSA} 1 \%$ and applied to sections and then incubated at $4{ }^{\circ} \mathrm{C}$ overnight. Sections were washed 3 times in PBS $(0.01 \mathrm{M})$ for 5 mins. Goat anti-rabbit TRITC secondary antibody diluted $1 / 1000$ in PBS was then applied to the sections and incubated for 2 hours at room temperature. Sections were then washed at $3 \times 10 \mathrm{~min}$ utes in PBS $(0.01 \mathrm{M})$ and mounted with DAKO hardset fluorescent mounting media. Positive fluorescent staining was captured using an Olympus BX61 microscope (objective $x 2$ ) and Microfire Optronics digital camera.

\section{Statistics}

The data are presented as mean \pm S.D., and were analyzed using a One way repeated measures Analysis of Variance (ANOVA), non parametric Dunnet post test 


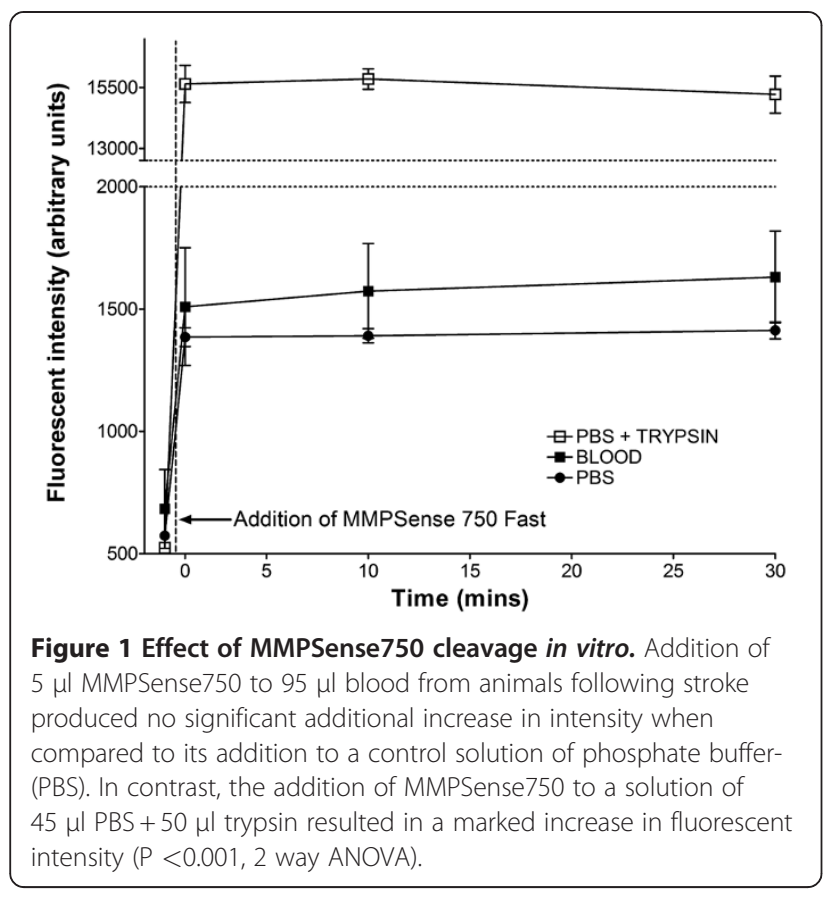

and Kruskal Wallis ANOVA for Ranks for left right comparison of continuous data. Differences were considered significant at $\mathrm{p}<0.05$.

\section{Results}

MMPSense750 has a significant baseline signal and is unaffected by blood from stroke animals examined in vitro

The addition of MMPSense750 to a sample of PBS resulted in an immediate increase in the NIR fluorescent intensity detected indicating that the optical solution contains unconjugated probe that is optically active and provides a background level of grey scale fluorescence intensity (Figure 1). The addition of MMPSense750 to blood from stroke animals did not result in a substantial additional increase in gray scale intensity relative to PBS suggesting that there is little activation of the probe following the administration of blood (Figure 1). The addition of $5 \mu \mathrm{l}$ PBS instead of MMPSense750 to blood did not result in a significant change in intensity (data not shown). However, the addition of MMPSense750 to $45 \mu \mathrm{l} \mathrm{PBS}+50 \mu \mathrm{l}$ trypsin resulted in a marked increase in fluorescent signal indicative of optical activation of the probe by proteolytic cleavage with trypsin.

\section{MMPSense750 is activated in early ischemia reperfusion}

The cortical CBF change in all animals successfully reached the set threshold $(>70 \%$ decrease from baseline). The mean LDF reduction for each group was: early ischemia reperfusion $2 \mathrm{HR}(84 \%, \mathrm{SD} 3.6)$, early ischemia reperfusion with the induction of hypothermia $\left(33^{\circ} \mathrm{C}\right)$

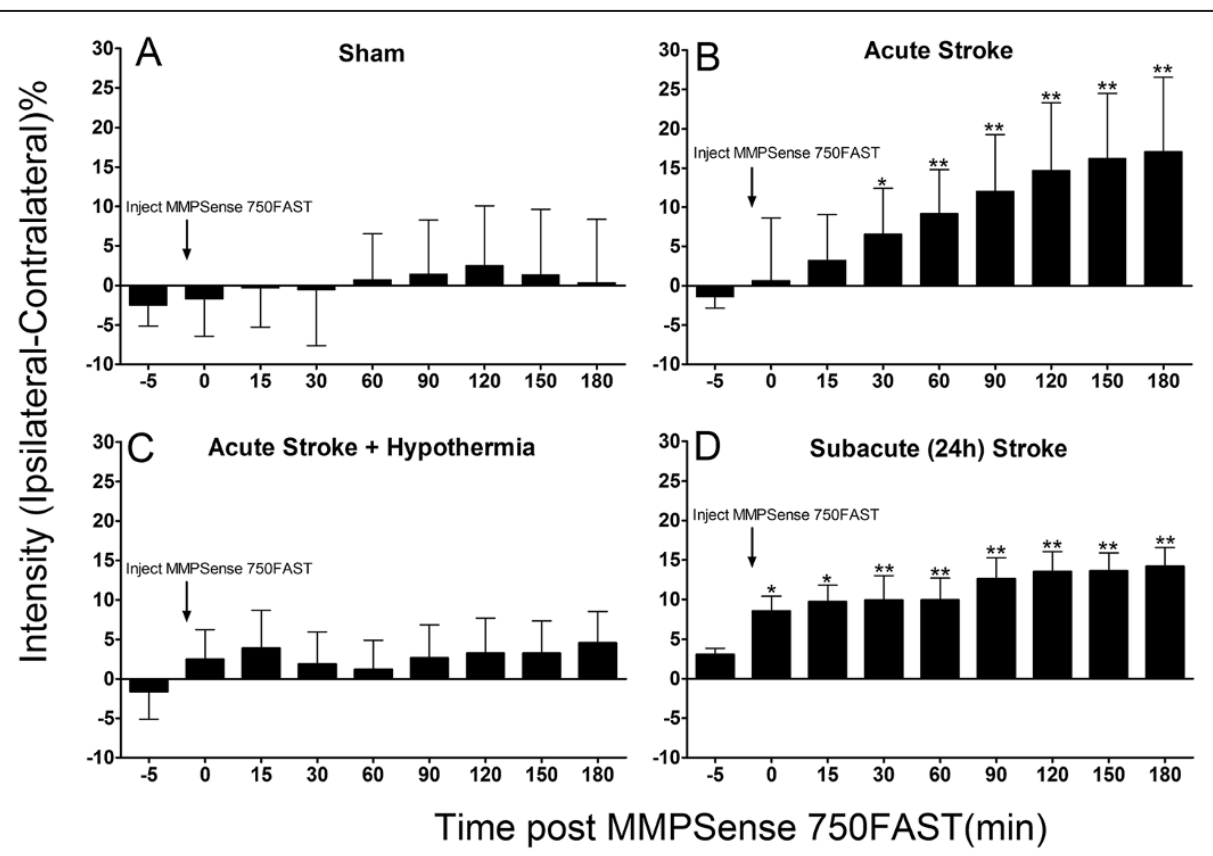

Figure 2 MMPSense750 activation at acute (2 hour) and subacute (24 hour) time points after reperfusion from MCAO. MMPSense750 injection in sham animals (A) resulted in no significant ipsilateral versus contralateral differences in fluorescent intensity whereas with acute MCAO reperfusion $(\mathbf{B})$, there were significant differences in fluorescent intensity by 30 minutes $(P<0.05)$ post injection that persisted until 180 minutes. At 24 hours post ischemia there were immediate ipsilateral-contralateral differences following probe injection that persisted for all the times examined. The increase in ipsilateral signal intensity was attenuated by hypothermia (D). ${ }^{*} \mathrm{P}<0.05,{ }^{* *} \mathrm{p}<0.01$ comparison to pre-injection value (One way repeated measures ANOVA, non parametric Dunnet post test). 

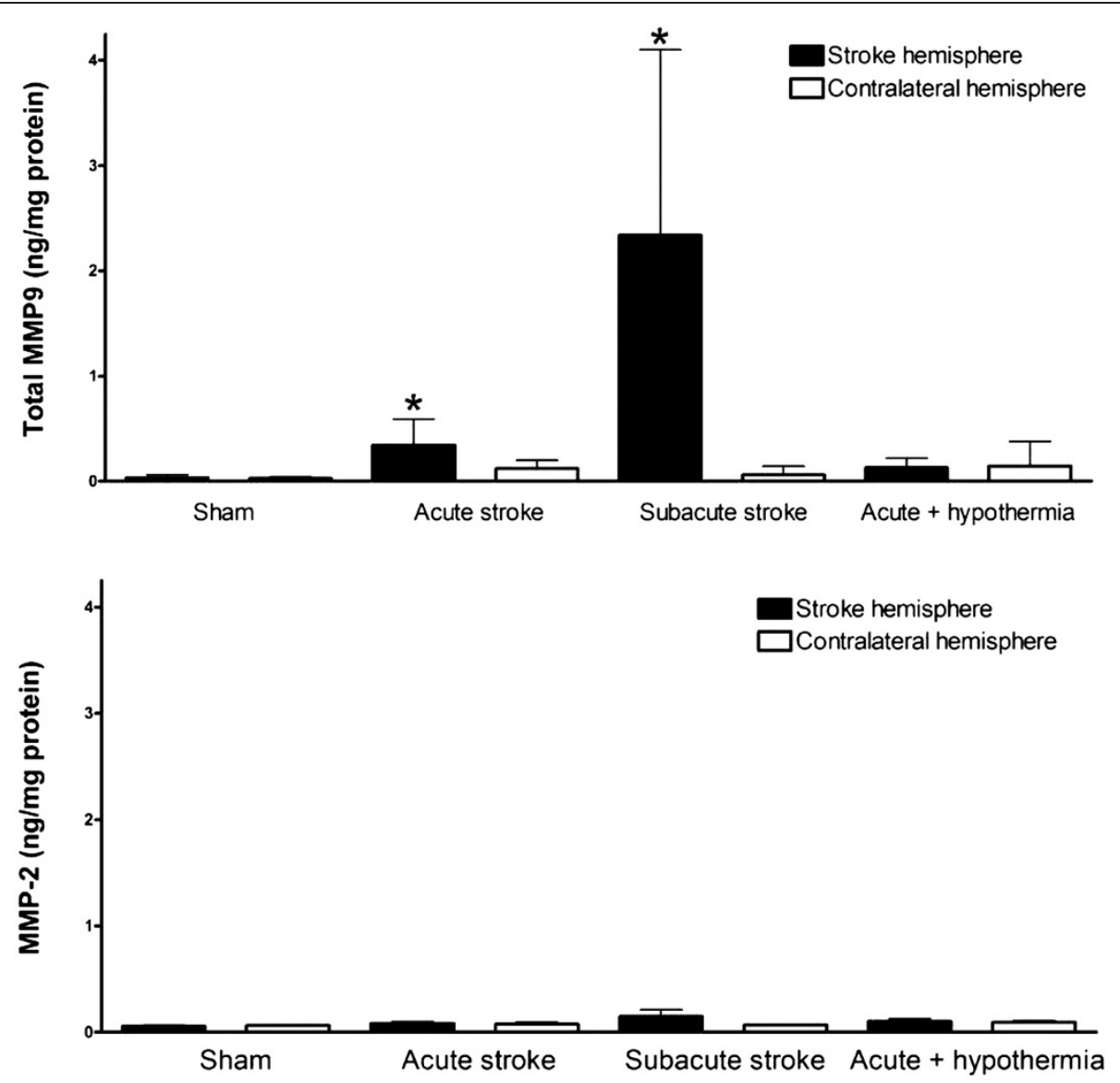

Figure 3 MMP 9 and MMP2 expression following early (5hour) and subacute (24 hour) ischemia reperfusion. An increased level of MMP expression was apparent at 5 hours and 24 hours post ischemia reperfusion ( ${ }^{*} \mathrm{P}<0.05$; different from corresponding Sham surgery control; Kruskal Wallis ANOVA and Dunn's test). An increase in MMP 2 was not observed either in early (5 hr) or subacutely (24 hour) ischemic reperfusion.

(81\%, SD2.8), and ischemia reperfusion at 24 hours (93\%,SD5.9). There was no statistical difference between animal groups $(\mathrm{P}=0.33)$.

Two hours after the onset of reperfusion post ischemia, administration of MMPSense750 resulted in an increase of NIR fluorescent intensity (Figure 2.B P <0.05) levels at 30-180 minutes following injection not seen in sham controls (Figure 2A). Acute stroke combined with hypothermia reduced MMPSense750NIR intensity levels (Figure 2C). The optical intensity increase persisted up to the final recording at 5 hours of ischemia reperfusion. Similar results were observed following injection of MMPSense750 at 24 hour stroke, the difference being that the initial rate of change and maximal optical intensity occurred earlier within minutes of administering the MMPSense 750 (Figure 2D) indicative of the presence of substantial quantities of MMPs/proteases in the cortex resulting in an abrupt activation of the MMPSense750 rather than a steady rise in MMPs or intensity at the acute time point (Figure $2 \mathrm{~B}$ ). No statistical difference in optical intensity between the left and right hemisphere was seen in sham operated animals or in animals with hypothermia induced immediately after 30 minutes of MCAO (Figure $2 \mathrm{~A}, \mathrm{C}$ ).

\section{MMP-9 protein is predominately upregulated in early} ischemia reperfusion and at 24 hours

Quantification of total MMP-9 protein from brain homogenates using ELISA revealed a predominant increase in MMP-9 levels at 5 and 24 hours post ischemia reperfusion (Figure 3), with higher MMP-9 levels detected in the stroke hemisphere during a subacute stroke versus and acute stroke (Figure 3). This increase in MMP-9 levels in acute stroke were found to be attenuated by hypothermia $(\mathrm{P}<0.05)$ (Figure 3$)$. Changes in MMP-2 protein expression were not detected at either time point (Figure 3). This increase in MMP-9 was confirmed with positive immunofluoresence staining for MMP-9 within the ipsilateral stroke hemisphere and corresponding reductions of such staining with hypothermia (Figure 4). Changes corresponding to these altered MMP-9 levels were visualized in vivo using NIR 


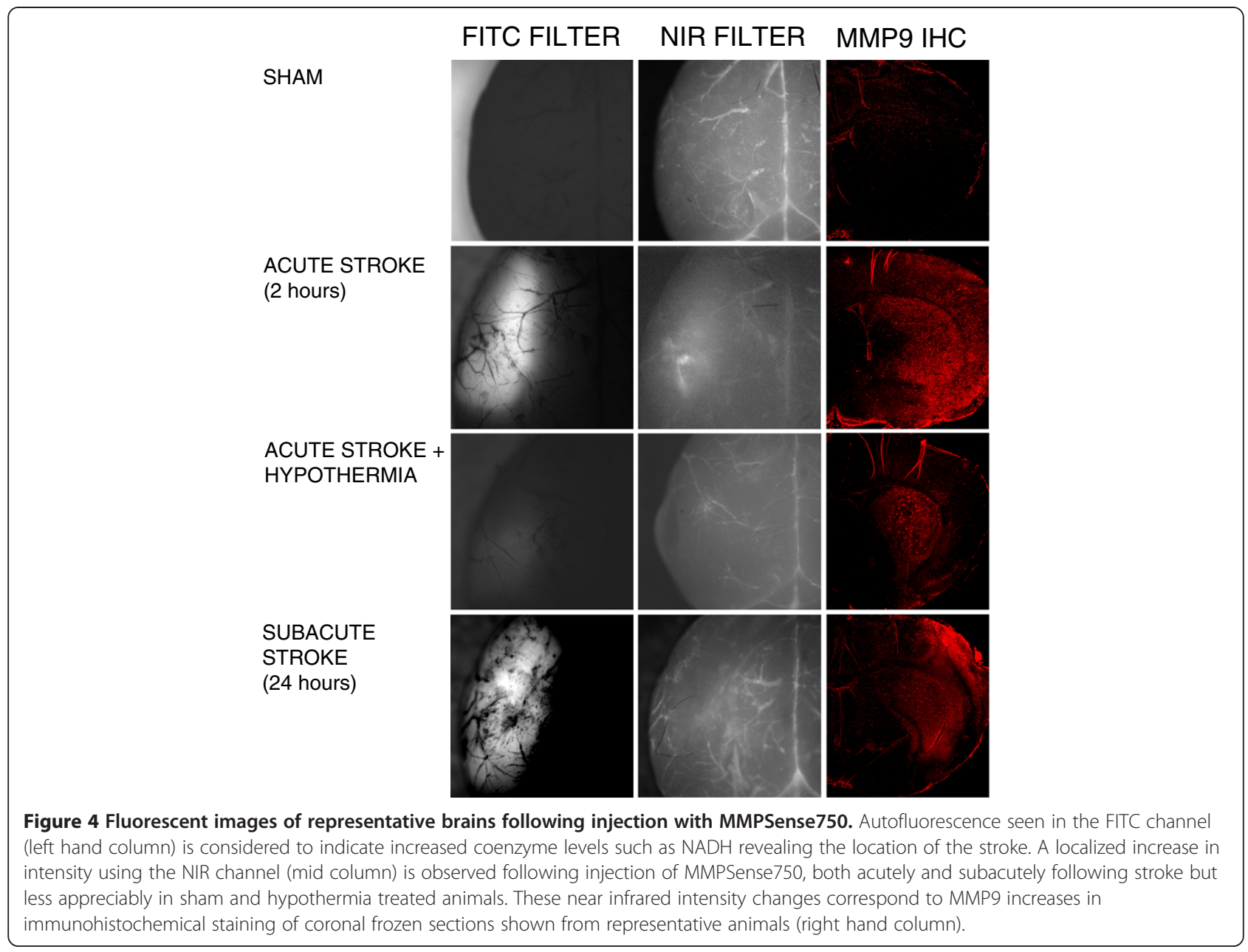

fluorescence imaging of the activated MMPSense750 in the stroke region (Figure 4) compared to sham controls. In animals maintained hypothermic for 5 hours following stroke, there was an attenuation of the activated fluorescent signal intensity (Figure 4).

\section{Discussion}

This study reports a novel early up-regulation of MMP9 in ischemic reperfusion. The acute in-vivo detection of MMP upregulation following ischemic stroke has not been reported previously. Importantly, we describe an innovative technique by which the increase in MMP-9 levels can be imaged in-situ within the first few hours of ischemia reperfusion using NIR fluorescent imaging and an activatable optical probe MMPSense750. Furthermore, we demonstrate that hypothermia attenuates the optical intensity increase in MMPSense750 and the increase in MMP-9 protein expression among the broad range of MMP's potentially detected by MMPSense750, (e.g. MMP 2, 3, 7, 9, 12, and 13). Our data provides evidence that MMPSense750 can detect activity of MMPs in-vivo and taken together our findings support the proof of concept that NIR fluorescent imaging can be used in situ to assess the mechanisms involved in cerebral ischemia.

The expression of MMP-9 corresponded well with the spatial and temporal activation of MMPSense750. The time dependent activities of MMPs have been previously well documented [20,21]. Of the two MMPs - 9 and 2 that are consistently reported to be up-regulated in ischemic stroke, we found MMP-9 to be the most strongly expressed in early ischemia reperfusion and later at 24 hours post reperfusion. The increased activity of the MMPSense750 sense probe was evident regionally and temporally in both the infarct and periinfarct regions when compared with the regions of endogenous FITC fluorescence considered to represent areas of predominantly increased NADH within the ischemic lesion $[18,19]$. The early increase in NIR optical intensity would suggest that MMPSense750 is being activated by increased MMP activity either at the intraluminal surface by activated leukocytes or at the basal lamina [16]. It is important to note that when using this probe it is necessary to control for presence of 
unconjugated probe and potential systemic activation of the probe in vivo, because when we added MMPSense750 to PBS fluorescent intensity immediately increased and MMPSense750 administered to sham operated mice produced immediate low level near infrared fluorescence intensity increases equally distributed over both hemispheres.

Non invasive imaging of MMP activity remains a clinically important unmet need. In a recent exploratory study, a NIR fluorescent probe with excitation emission in the $680 \mathrm{~nm}$ wavelength range was used to detect MMP activity in an animal model of stroke using NIRF imaging accompanied by magnetic resonance and radioisotope imaging techniques [12]. In this study, the utility of this $680 \mathrm{~nm}$ probe was investigated only at 24 hours after ischemia. For optimal clinical application, it is important to have an activatable probe that can also detect early MMP upregulation following cerebral ischemia and the present results demonstrate MMPSense750 can detect earlier acute changes in $\mathrm{MMP} /$ protease activity.

There were recognized limitations of our study. We used gelatin zymography to detect MMP-2 and 9 in brain tissues, and the levels of these MMPs were too low to be detected by this technique suggesting the importance of our imaging method, which is sensitive enough to detect low levels of MMP activity. The fact that we were not able to detect large quantities of pro-MMP-9 would suggest that the optical changes measured included activated MMP-9. Clearly also possible is that other MMP's contributed to the MMPSense intensity increases observed. Furthermore, our observation that trypsin activates MMPSense750 in vitro supports the conclusion that this probes activation can be induced by other proteases such as serine proteases in addition to MMPs. Another limitation was our inability to readily image NIR fluorescent changes though the skull. Non invasive detection is theoretically possible with NIR probes but considering the small magnitude of the changes observed we believe this would need substantial technical improvements with advances in non-invasive imaging systems and probe sensitivity. In addition to the small changes in signal intensity, the need for background correction for this particular probe may limit its clinical applicability.

A novel component of this study was the in vivo demonstration that moderate hypothermia induced after ischemia reperfusion reduced both the NIR optical signal intensity and also the level of MMP-9 protein detected. Previous studies from MMP gene knockout mice and those using MMP pharmacological inhibitors suggest that the MMPs may be attractive therapeutic targets for stroke [22-24]. It has been demonstrated that moderate hypothermia $\left(32-34^{\circ} \mathrm{C}\right)$ protects the basal lamina, reduces infarct volume and hemorrhage, and reduces MMP-9 [25,26]. However, emerging data now also suggests that some aspects of MMP activity during the delay of neuroinflammatory response may contribute to re-modeling in stroke recovery [27]. Therefore, defining the time dependent relationship of MMP activation has been increasingly emphasized and timing of therapeutic strategies require detailed refinement to avoid the potential theoretical deleterious effects of MMP inhibition on stroke remodeling. In this capacity the use of experimental tools such as near NIR fluorescent probes and NIR imaging may become invaluable both in pre clinical and clinical testing.

\section{Conclusions}

This study shows that matrix-metalloproteinase upregulation in ischemia reperfusion can be imaged acutely invivo with NIRF using MMPSense750. Hypothermia attenuated both the optical increase in intensity after MMPSense750 and the increase in MMP-9 protein expression supporting the proof of concept that NIRF imaging using MMPSense can be used to assess potential therapeutic strategies for stroke treatment.

\section{Competing Interests \\ The author(s) declare that they have no competing interests.}

\section{Authors' Contributions}

PB conceptualized the study design, was involved in data analysis, coordinated all aspects of the study, wrote the manuscript and critically appraised the manuscript. DR performed the in vivo stroke animal studies and contributed to data acquisition and analysis. SA was involved in data acquisition and tissue processing as well as the critical appraisal of the manuscript. UT was involved in the conceptualization of the study, data analysis and acquisition, in addition to contributing to critical appraisal and writing of the manuscript. All authors read and approved the final manuscript.

\section{Acknowledgements}

This study was supported by a grant from the Heart and Stroke Foundation of Alberta. P.A.B. was supported by the Alberta Heritage Foundation for Medical Research and the Heart and Stroke Foundation of Canada. The authors would also like to thank the National Research Council of Canada for their help with the many technical aspects of the study.

\section{Author details}

${ }^{1}$ Department of Clinical Neurosciences, Experimental Imaging Centre and Hotchkiss Brain Institute, Faculty of Medicine, University of Calgary Calgary, Canada. ${ }^{2}$ BBD West, National Research Council, Canada. ${ }^{3}$ Experimental

Imaging Centre, B153, 3330 Hospital Dr NW, University of Calgary Calgary, AB T2N 2T9, Canada.

Received: 23 March 2012 Accepted: 18 June 2012

Published: 28 June 2012

\section{References}

1. Del Zoppo GJ: The neurovascular unit, matrix proteases, and innate inflammation. Ann N Y Acad Sci 2010, 1207:46-49.

2. Yong WW, Krekoski CA, Forsyth PA, Bell R, Edwards DR: Matrix metalloproteinases and diseases of the CNS. Trends Neurosci 1998, 21:75-80.

3. Rosenberg GA, Navratil M, Barone F, Feuerstein G: Proteolytic cascade enzymes increase in focal cerebral ischemia in rat. $J$ Cereb Blood Flow Metab 1996, 16:360-366.

4. Sumii T, Lo EH: Involvement of matrix metalloproteinase in thrombolysisassociated hemorrhagic transformation after embolic focal ischemia in rats. Stroke 2002, 33:831-836. 
5. Ramos-Fernandez M, Bellolio MF, Stead LG: Matrix metalloproteinase-9 as a marker for acute ischemic stroke: a systematic review. J Stroke Cerebrovasc Dis 2011, 20:47-54.

6. MacLellan CL, Clark DL, Silasi G, Colbourne F: Use of prolonged hypothermia to treat ischemic and hemorrhagic stroke. J Neurotrauma 2009, 26:313-323.

7. Choi HA, Badjatia N, Mayer SA: Hypothermia for acute brain injurymechanisms and practical aspects. Nat Rev Neurol 2012, 8(4):214-22

8. Pires PW, Rogers CT, McClain JL, Garver HS, Fink GD, Dorrance AM: Doxycycline, a matrix metalloprotease inhibitor, reduces vascular remodeling and damage after cerebral ischemia in stroke-prone spontaneously hypertensive rats. Am J Physiol Heart Circ Physiol 2011, 301 (1):H87-97.

9. Rodriguez-Gonzalez R, Sobrino T, Rodriguez-Yanez M, Millan M, Brea D,

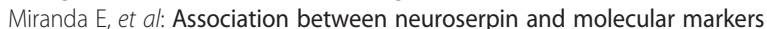
of brain damage in patients with acute ischemic stroke. J Trans/ Med 2011, 9:58

10. Chaudhry K, Rogers R, Guo M, Lai Q, Goel G, Liebelt B, et al: Matrix metalloproteinase-9 (MMP-9) expression and extracellular signalregulated kinase 1 and 2 (ERK1/2) activation in exercise-reduced neuronal apoptosis after stroke. Neurosci Lett 2010, 474:109-114.

11. Yoon SM, Myung SJ, Ye BD, Kim IW, Lee NG, Ryu YM, et al: Near-infrared fluorescence imaging using a protease-specific probe for the detection of colon tumors. Gut Liver 2010, 4:488-497.

12. Klohs J, Baeva N, Steinbrink J, Bourayou R, Boettcher C, Royl G, et al: In vivo near-infrared fluorescence imaging of matrix metalloproteinase activity after cerebral ischemia. J Cereb Blood Flow Metab 2009, 29:1284-1292.

13. Truettner JS, Alonso OF, Dalton DW: Influence of therapeutic hypothermia on matrix metalloproteinase activity after traumatic brain injury in rats. J Cereb Blood Flow Metab 2005, 25:1505-1516.

14. Baumann E, Preston E, Slinn J, Stanimirovic D: Post-ischemic hypothermia attenuates loss of the vascular basement membrane proteins, agrin and SPARC, and the blood-brain barrier disruption after global cerebral ischemia. Brain Res 2009, 1269:185-197.

15. Horstmann S, Koziol JA, Martinez-Torres F, Nagel S, Gardner H, Wagner S: Sonographic monitoring of mass effect in stroke patients treated with hypothermia. Correlation with intracranial pressure and matrix metalloproteinase 2 and 9 expression. J Neurol Sci 2009, 276:75-78.

16. Nagel S, Su Y, Horstmann S, Heiland S, Gardner H, Koziol J, et al: Minocycline and hypothermia for reperfusion injury after focal cerebral ischemia in the rat: effects on BBB breakdown and MMP expression in the acute and subacute phase. Brain Res 2008, 1188:198-206.

17. Barber PA, Hoyte L, Colbourne F, Buchan A: Temperature regulated model of focal ischemia in the mouse: a study with histopathological and behavioural outcomes. Stroke 2004, 35:1720-1725.

18. Kasischke KA, Lambert EM, Panepento B, Sun A, Gelbard HA, Burgess RW, et al: Two-photon NADH imaging exposes boundaries of oxygen diffusion in cortical vascular supply regions. J Cereb Blood Flow Metab 2011, 31:68-81.

19. Mayevsky A, Rogatsky GG: Mitochondrial function in vivo evaluated by $\mathrm{NADH}$ fluorescence: from animal models to human studies. Am J Physio/ Cell Physiol 2007, 292:C615-C640.

20. Chang DI, Hosomi N, Lucero J, Heo JH, Abumiya T, Mazar AP, et al: Activation systems for latent matrix metalloproteinase-2 are upregulated immediately after focal cerebral ischemia. J Cereb Blood Flow Metab 2003, 23:1408-1419.

21. Yang Y, Estrada EY, Thompson JF, Liu W, Rosenberg GA: Matrix metalloproteinase-mediated disruption of tight junction proteins in cerebral vessels is reversed by synthetic matrix metalloproteinase inhibitor in focal ischemia in rat. J Cereb Blood Flow Metab 2007, 27:697-709.

22. Dong X, Song YN, Liu WG, Guo XL: Mmp-9, a potential target for cerebral ischemic treatment. Curr Neuropharmacol 2009, 7:269-275.

23. Ponnampalam SN, Mayberg MR: Mediators of blood-brain barrier disruption and potential therapeutic interventions for protection of the barrier following focal ischemia. Clin Neurosurg 2004, 51:112-119.

24. Horstmann S, Kalb P, Koziol J, Gardner H, Wagner S: Profiles of matrix metalloproteinases, their inhibitors, and laminin in stroke patients: influence of different therapies. Stroke 2003, 34:2165-2170.

25. Burk J, Burggraf D, Vosko M, Dichgans M, Hamann GF: Protection of cerebral microvasculature after moderate hypothermia following experimental focal cerebral ischemia in mice. Brain Res 2008, 1226:248-255

26. Lee JE, Yoon YJ, Moseley ME, Yenari MA: Reduction in levels of matrix metalloproteinases and increased expression of tissue inhibitor of metalloproteinase- 2 in response to mild hypothermia therapy in experimental stroke. J Neurosurg 2005, 103:289-297.

27. Lo EH: A new penumbra: transitioning from injury into repair after stroke. Nat Med 2008, 14:497-500.

doi:10.1186/1471-2202-13-76

Cite this article as: Barber et al:: Infrared optical imaging of matrix metalloproteinases (MMPs) up regulation following ischemia reperfusion is ameliorated by hypothermia. BMC Neuroscience 2012 13:76.

\section{Submit your next manuscript to BioMed Central and take full advantage of:}

- Convenient online submission

- Thorough peer review

- No space constraints or color figure charges

- Immediate publication on acceptance

- Inclusion in PubMed, CAS, Scopus and Google Scholar

- Research which is freely available for redistribution

Submit your manuscript at www.biomedcentral.com/submit
C) Biomed Central 